\title{
Effect of inhaled fluticasone propionate on airway responsiveness in treatment-naive individuals - a lesser benefit in females
}

\author{
R.P. Convery*, D.N. Leitch*, C. Bromly*, R.J. Ward*, G. Bartlett**, D.J. Hendrick*,**
}

Effect of inhaled fluticasone propionate on airway responsiveness in treatment-naive individuals - a lesser benefit in females. R.P. Convery, D.N. Leitch, C. Bromly, R.J. Ward, G. Bartlett, D.J. Hendrick. C ERS Journals Ltd 2000.

ABSTRACT: A randomized double-blind placebo-controlled parallel group study with inhaled fluticasone propionate over 6 weeks, designed to quantify the beneficial effect on airway responsiveness, and so assess whether short pulses of intermittent prophylactic treatment might serve as an alternative means of managing mild asthma, is reported.

The 20-50-yr-old participants, who were recruited from an epidemiological study of the general population, had never knowingly received any regular treatment for asthma. Fluticasone propionate at the maximum recommended dose level $(2,000 \mu \mathrm{g}$ daily) and placebo were administered via metered-dose inhalers, and airway responsiveness was quantified conventionally by the provocative dose of methacholine causing a 20\% fall in forced expiratory volume in one second (FEV1) (PD20) at 2-week intervals during the treatment phase and at various intervals subsequently.

Compared with placebo fluticasone propionate was associated with a highly significant decrease in airway responsiveness (1.9 doublings of the geometric mean PD20), which was maximal at the end of the 6-week treatment period. No persisting benefit was detectable at the next measurement 2 weeks later, or thereafter. Multiple linear regression analysis showed that the magnitude of the fluticasone propionate effect was significantly greater in males than in females (3.2 versus 1.2 doublings respectively of the geometric mean PD20), but was uninfluenced by current smoking, age or FEV1.

In conclusion, in the absence of any possibility of tachyphylaxis, inhaled fluticasone propionate at this dose causes a steadily increasing improvement in airway responsiveness over a 6-week period, which is modified by sex but lost almost immediately on treatment cessation. Short pulses of intermittent prophylactic treatment would not, therefore, be useful as a means of managing mild asthma.

Eur Respir J 2000; 15: 19-24.

In an epidemiological study of a normal population of males and females many subjects were identified in whom airway responsiveness could be quantified but who had never knowingly received corticosteroid treatment, whether for asthma or other diseases, nor knowingly received any regular medication for asthma for $>3$ months [1]. The majority were not recognized to have (or to have had) asthma. Volunteers from among them were sought to evaluate the effect on airway responsiveness of inhaled fluticasone propionate, a potent topical steroid thought to have an enhanced benefit-risk ratio because of a high level of "first pass" hepatic metabolism and low oral bioavailability [2].

The aim of this study was to quantify the effect of 6 weeks treatment at the maximum recommended dose by its peak and duration over the following 20 weeks. It was wondered whether short "pulses" of intermittent prophylactic treatment might offer an alternative means of

$\overline{\text { For editorial comments see page } 3}$
*Dept of Respiratory Medicine, Royal Victoria Infirmary, University of Newcastle upon Tyne, Newcastle upon Tyne, UK. **Joint Depts of Epidemiology and Biostatistics and Occupational Health, McGill University, Montreal, Canada.

Correspondence: D.J. Hendrick

Dept of Respiratory Medicine

Royal Victoria Infirmary

Queen Victoria Road

Newcastle upon Tyne

NE1 4LP

UK.

Fax: 441912275224

Keywords: Airway responsiveness asthma

corticosteroids

fluticasone

sex

smoking

Received: March 31999

Accepted after revision October 161999

This study was supported by GlaxoWellcome Group Research. management of mild asthma (or a means of preventing 'subclinical asthma' from becoming symptomatic) if the initial effect was sufficiently strong and sufficiently prolonged. The secondary aims were to assess the possible influence of sex and smoking on any treatment effect, since female sex and smoking are both associated with heightened disease severity [3-6].

\section{Methods}

\section{Airway responsiveness}

Airway responsiveness was measured using methacholine inhalation tests with optimal precision, and quantified conventionally by the provocative dose of methacholine causing a $20 \%$ fall in forced expiratory volume in one second (FEV1) (PD20) [7]. A failure to produce a $20 \%$ fall in FEV1 after the final dose following a doubling cumulative dose protocol (range 3,125-6,400 $\mu \mathrm{g}$ ) constitutes a negative test. With the test system used, PD20 of $<200 \mu \mathrm{g}$ are generally associated with other clear evidence of 
active asthma, whereas PD20 of $>1,000 \mu \mathrm{g}$ are generally not associated with asthmatic symptoms. PD20 in the range 200-1,000 $\mu \mathrm{g}$ represent a "grey" zone in which some subjects have mild symptomatic asthma and others do not. PD20 are generally repeatable within one doubling dose under ideal conditions or 1.6 doubling doses. Thus the coefficient of repeatability is of the order of 2-3.

\section{Subjects}

It was attempted to recruit 15 subjects into each of four subgroups determined by sex and smoking status, with the expectation that 10 would complete the study in each category. Calculations from stored data from repeated methacholine tests in other subjects suggested that this would provide $80 \%$ power at a $5 \%$ significance level for detecting a clinically meaningful difference in fluticasone propionate benefit between females and males (2.8-fold difference in geometric mean PD20, two tailed), or between nonsmokers and smokers (2.5-fold difference, one tailed). Volunteers were chosen to have PD20 of $<1,600 \mu \mathrm{g}$ from two baseline methacholine tests conducted within 2 weeks but separated by $48 \mathrm{~h}$.

Participants were also chosen to be aged 20-50 yrs. Only those in one of two smoking categories over the preceding 2 yrs were accepted: "nonsmokers" who had not smoked, and "smokers" who had smoked at least five cigarettes daily. None were taking $\beta$-blocking or antimuscarinic drugs, and none were using aspirin or other nonsteroidal anti-inflammatory agents other than at a constant dose. None had had any respiratory tract infection requiring antibiotics within the preceding 4 weeks, an uncontrolled systemic disease or a history of alcohol abuse. All were able to use a metered-dose inhaler (MDI) adequately and all gave written informed consent to participate. The investigation was approved by the local Ethics Committee and conducted according to the Declaration of Helsinki.

\section{Study medication}

Placebo or fluticasone propionate at the maximum dose recommended for regular use $(2,000 \mu \mathrm{g}$ daily) was administered each morning for six weeks via seemingly identical pressurized MDIs at seemingly identical doses.

\section{Protocol}

The study was carried out in a randomized doubleblind placebo-controlled parallel group fashion, stratified by sex and smoking habit. Fully compliant participants visited the research laboratory on eight occasions (study visits V0-7). The initial recruitment visit (V0) during the pretreatment phase of the study was used to obtain baseline data concerning: demography; smoking; clinical examination; weight; height; spirometry; methacholine responsiveness; and expired carbon monoxide concentration. A placebo MDI was then prescribed for all participants in order to familiarize them with its use. At the second pretreatment phase visit (V1) and at all subsequent visits during the treatment phase (V2-4) and follow-up phase (V5-7), spirometry and the methacholine test were repeated.
The pretreatment phase was completed with 1-2 weeks for each individual participant. Randomization for treatment with placebo or fluticasone propionate was carried out at V1 for subjects who met the inclusion criteria and were willing to proceed with the investigation. Treatment was commenced immediately following $\mathrm{V} 1$, and was given for 6 weeks exactly. The effects were evaluated during the treatment phase after 2, 4 and 6 weeks of treatment (V2, 3 and 4). Further evaluations were made during the follow-up phase 8,12 and 26 weeks after V1 (V5, 6 and 7). These were 2, 6 and 20 weeks after completion of the treatment phase.

\section{Statistical analysis}

SAS 6.0 (SAS Institute, Cary, NC, USA) was used for statistical analyses. Because the methacholine was administered using a doubling cumulative dose protocol, all PD20 were log-transformed in order to obtain a normal distribution. Categorical variables were compared using the Chi-squared test and continuous variables were compared using Student's t-test. Changes in PD20 from baseline (geometric mean of V0 and V1; VBPD20) were compared using a paired t-test. A Bonferroni correction was used with these Chi-squared tests and t-tests to allow for multiple testing $(\alpha=0.01)$. Multiple linear regression analysis was used to model the effect of treatment on $\ln P D 20$ at visits V2-7 while controlling for the effects of sex, smoking, age, FEV1 and VBPD20. Possible interactions between treatment and sex, smoking, age and FEV1 were tested at each visit to establish whether the treatment effect depended on these variables.

\section{Results}

\section{Subjects}

A total of 52 subjects completed the pretreatment phase of the study satisfactorily and reattended for at least one (but non necessarily all) of the treatment phase visits. All but 13 attended all eight visits. The distribution by treatment group, sex and smoking category is given in table 1, with demographic data and mean baseline FEV1 and lnPD20. There were no important aberrations in distribution between the treatment and sex/smoking groupings, although females were necessarily shorter than males and had lower FEV1, and smokers had lower FEV1 than nonsmokers. There were no significant differences in PD20 between the treatment groups or sexes at baseline.

\section{Expired levels of carbon monoxide}

The one missing value (in a nonsmoker) was replaced by the nonsmoking mean. A level of $\sim 9$ parts per million (ppm) has been suggested as the diagnostic threshold for separating nonsmokers from smokers [8]. Only one of the 27 nonsmokers yielded a higher value (13 ppm) and only one of the 25 smokers a lesser value $(7 \mathrm{ppm})$. The mean \pm SD levels for the nonsmokers and smokers were clearly and significantly different at $4.8 \pm 2.1 \mathrm{ppm}$ and $19.5 \pm 7.7$ ppm respectively $(\mathrm{p}<0.0001)$. 
Table 1. - Distribution of smoking category and demographic and baseline data by sex and treatment

\begin{tabular}{|c|c|c|c|}
\hline & Placebo & $\begin{array}{l}\text { Fluticasone } \\
\text { propionate }\end{array}$ & Total \\
\hline Subjects & 26 & 26 & 52 \\
\hline Females & 15 & 16 & 31 \\
\hline Nonsmokers & 6 & 7 & 13 \\
\hline Smokers & 9 & 9 & 18 \\
\hline Age yrs & $35 \pm 7$ & $33 \pm 10$ & $34 \pm 8$ \\
\hline Height m & $1.67 \pm 0.04$ & $1.64 \pm 0.06$ & $1.66 \pm 0.05$ \\
\hline $\mathrm{BMI} \mathrm{kg} \cdot \mathrm{m}^{-2}$ & $24.6 \pm 5.8$ & $23.7 \pm 3.0$ & $24.1 \pm 4.5$ \\
\hline VBFEV $1 \%$ pred & $101.7 \pm 13.8$ & $100.9 \pm 11.4$ & $101.3 \pm 12.4$ \\
\hline VBlnPD20 $\mu \mathrm{g}$ & $5.63 \pm 1.31$ & $5.68 \pm 0.83$ & $5.65 \pm 1.07$ \\
\hline Males & 11 & 10 & 21 \\
\hline Nonsmokers & 7 & 7 & 14 \\
\hline Smokers & 4 & 3 & 7 \\
\hline Age yrs & $34 \pm 8$ & $28 \pm 7$ & $31 \pm 8$ \\
\hline Height $m$ & $1.79 \pm 0.06$ & $1.82 \pm 0.07$ & $1.8 \pm 0.07$ \\
\hline $\mathrm{BMI} \mathrm{kg} \cdot \mathrm{m}^{-2}$ & $25.0 \pm 1.2$ & $22.5 \pm 3.2$ & $23.9 \pm 2.6$ \\
\hline VBFEV $1 \%$ pred & $96.5 \pm 13.3$ & $106.0 \pm 13.9$ & $101.1 \pm 14.1$ \\
\hline VBlnPD20 $\mu \mathrm{g}$ & $5.26 \pm 1.40$ & $5.41 \pm 1.10$ & $5.33 \pm 1.23$ \\
\hline
\end{tabular}

Data are presented as $\mathrm{n}$ or mean \pm SD. BMI: body mass index; VB: mean at baseline (V0 and V1) (forced expiratory volume in one second (FEV1): arithmetic mean; provocative dose of methacholine causing a $20 \%$ fall in FEV1 (PD20): geometric mean).

Baseline forced expiratory volume in one second and provocative dose of methacholine causing a $20 \%$ fall in forced expiratory volume in one second

The arithmetic mean (SD) baseline (V0 and V1) FEV1 (VBFEV1) was $3.61 \pm 0.89 \mathrm{~L}$, which was $101 \%$ of the predicted value, and the VBPD20 was $251 \mu \mathrm{g}$. The corresponding values for the two treatment groups and the sex subgroups are given with SD in table 1 .

\section{Change in forced expiratory volume in one second}

The numbers of participants at each of the V2-7 differed (table 2), and so the mean FEV1 are not directly comparable. No significant changes occurred at any of the V2-7 in either the placebo or the fluticasone propionate group from the respective baseline values.

Table 2. - Geometric provocative dose of methacholine causing a $20 \%$ fall in forced expiratory volume in one second (PD20) at each study visit compared with the respective baselines (VB) by treatment group

\begin{tabular}{lcccccc}
\hline & V2 & V3 & V4 & V5 & V6 & V7 \\
\hline $\begin{array}{l}\text { Subjects } \mathrm{n} \\
\text { Placebo }\end{array}$ & 52 & 47 & 48 & 46 & 45 & 43 \\
Subjects $\mathrm{n}$ & 26 & 24 & 25 & 25 & 25 & 23 \\
VB $\mu \mathrm{g}$ & 238 & 230 & 240 & 240 & 240 & 215 \\
V2-7 $\mu \mathrm{g}$ & 262 & 269 & 294 & 290 & 368 & 351 \\
p-value & NS & NS & NS & NS & 0.01 & 0.0003 \\
Fluticasone & & & & & & \\
Subjects n & 26 & 23 & 23 & 21 & 20 & 20 \\
VB $\mu \mathrm{g}$ & 264 & 245 & 236 & 266 & 262 & 272 \\
V2-7 $\mu \mathrm{g}$ & 481 & 569 & 1111 & 378 & 472 & 419 \\
p-value & 0.003 & 0.002 & 0.0001 & NS & $0.03^{*}$ & $0.04^{*}$ \\
\hline
\end{tabular}

*: NS with Bonferroni correction. V2-4: treatment phase; V5-7: follow-up phase.
Change in provocative dose of methacholine causing a $20 \%$ fall in forced expiratory volume in one second

For five participants (all in the fluticasone propionate group), negative results were obtained from methacholine tests at the end of the treatment phase (V4). For these, a PD20 of $6,401 \mu \mathrm{g}$ was assigned, which is likely to have underestimated the true increase in PD20 in the subjects concerned. Table 2 shows the changes in geometric mean PD20 from the respective baselines at each of V2-7. There were no changes in the placebo group during the treatment phase, but, in the fluticasone propionate group, the PD20 increased steadily with increasing significance. The difference in geometric mean PD20 between the placebo and fluticasone propionate groups was not significant at V2 (262 versus $481 \mu \mathrm{g})$, was borderline significant at V3 with the Bonferroni correction (269 versus $569 \mu \mathrm{g}, \mathrm{p}<0.05)$ and was very significant at V4 (294 versus $1,111 \mu \mathrm{g}, \mathrm{p}<0.003)$. The differences during the follow-up phase (V5, 6 and 7) were not significant, indicating that the effect of fluticasone propionate did not persist even to the first PD20 measurement 2 weeks after treatment was discontinued. In parallel, no significant change was noted at V5 from baseline in either the placebo or the fluticasone propionate group. There were, however, mild but significant (or borderline significant) changes from baseline in both groups at V6 and 7 (i.e. 12 and 26 weeks after V1), indicating a secular effect over the time-course of the study. This amounted to less than one doubling of the geometric mean PD20.

\section{Peak change and duration of effect}

Data from the 52, 47 and 48 subjects who attended at V2, 3 and 4 respectively showed a steadily increasing benefit from fluticasone propionate, but not placebo, throughout the 6-week period of treatment (fig. 1). There was no persisting benefit once treatment was discontinued, and so maximum benefit was observed at V4. A fundamentally similar pattern was noted among the 39 subjects who attended all of V0-7.

\section{Multiple linear regression models}

Initials models showed no effect of smoking, age or FEV1 on PD20 at V2-7 after allowing for the VBPD20 (i.e. these variables were not associated independently with change in PD20). Nor were there any interactions between these variables and treatment (i.e. they did not modify the effect of fluticasone propionate). They were consequently removed from the later and definitive models. When controlling for sex and VBPD20 (which did exert significant influence), the effect of fluticasone propionate was marginally nonsignificant at V2 $(\mathrm{p}<0.06)$ but very significant at V3 $(\mathrm{p}<0.005)$ and $4(\mathrm{p}<0.001)$. The effect was maximal at V4, but it was nonsignificant at all subsequent posttreatment visits, V5-7.

The VBPD20 was, as expected, a significant predictor of PD20 at all subsequent visits $(\mathrm{p}<0.0001)$. The model also demonstrated a significant treatment/sex interaction, indicating that fluticasone propionate exerted a greater effect in males than in females, although sex alone exerted no 

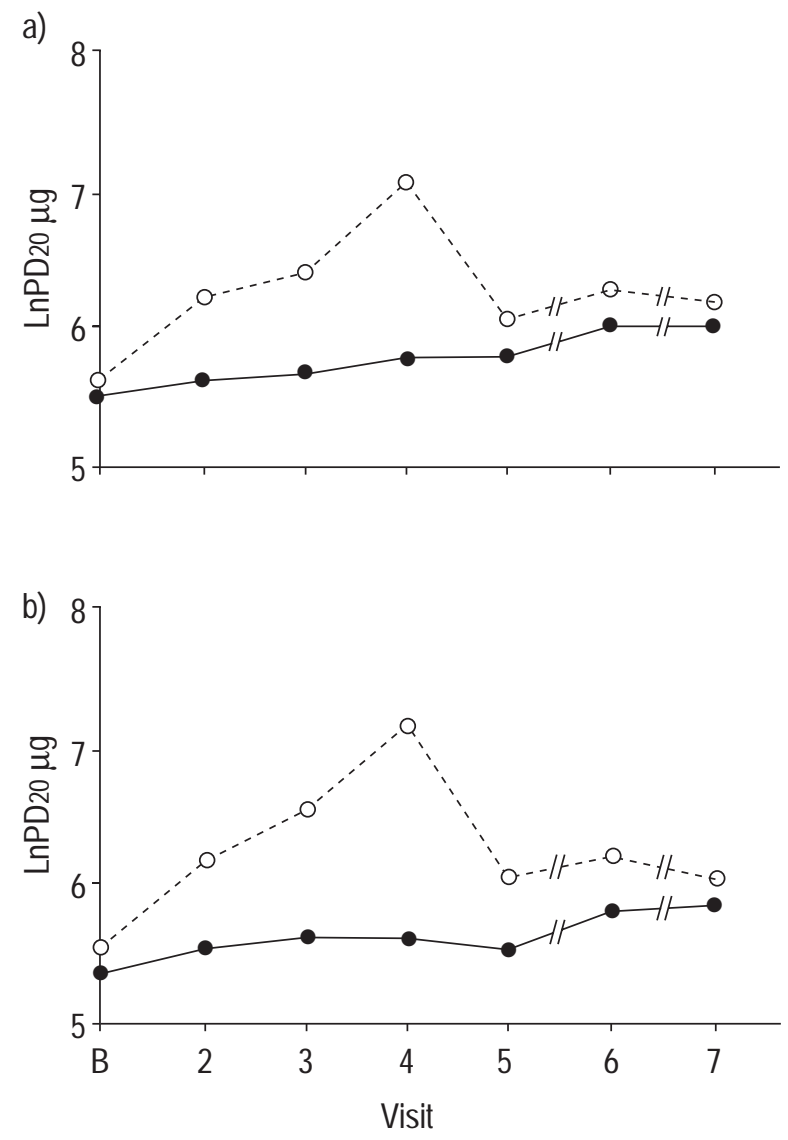

Fig. 1. - Geometric mean provocative dose of methacholine causing a $20 \%$ fall in forced expiratory volume in one second (PD20) at each visit (V) for: a) all participants; and b) the 39 participants attending all study visits $(\bigcirc$ : fluticasone propionate $2,000 \mu \mathrm{g}$ daily; 0 : placebo). Vs took place (with number of participants (a) in parentheses) at: baseline (B), 0 weeks ( $\mathrm{n}=52)$; V2, 2 weeks $(\mathrm{n}=52) ; \mathrm{V3}, 4$ weeks $(\mathrm{n}=47)$; V4, 6 weeks $(n=48) ;$ V5, 8 weeks $(n=46) ;$ V6, 12 weeks $(n=45) ;$ V7, 26 weeks $(n=43)$.

influence. Thus at V4, there were 1.2 doublings in geometric mean PD20 from baseline in females, which could be attributed to fluticasone propionate and an additional 2.0 doublings in males $(\mathrm{p}<0.04)$. This is equivalent to a 2.3 fold increase in the VBPD20 in females and an overall 9.0 fold increase in males. In order to estimate the net effect of fluticasone propionate in the study population as a whole, a final analysis ignored the effect of the treatment/sex interaction, and assessed only the effects of treatment and the VBPD20 on PD20 at V4. Fluticasone propionate was associated with 1.9 doublings (3.8 fold increase) of PD20 from baseline $(\mathrm{p}<0.0002)$.

Three of the participants, all randomized to the fluticasone propionate group (two male, one female, all nonsmokers), showed particularly marked improvements in airway responsiveness, with increases in PD20 from baseline to V4 exceeding 20 fold (4.3 doublings). No obvious technical problems were detected, and their serial PD20 were consistent with a genuine response to fluticasone propionate treatment. Furthermore, changes in PD20 over similarly wide ranges and similarly short periods have been noted in individuals with emergent occupational asthma in and away from the workplace, and in conducting specific inhalation provocation tests [9-11]; SAS "diag- nostics" testing did not identify an statistical outliers. Nevertheless, the influence of these participants was assessed by excluding their data and rerunning the analyses. The effect of fluticasone propionate continued to be significant ( 0.9 doublings in geometric mean PD20 in females, $\mathrm{p}<0.04)$, and a significant treatment/sex interaction favouring males was still evident (an additional 1.4 doublings in males, $\mathrm{p}<0.04$ ). There were thus significant increases of 1.8 and 5.0 fold overall in the females and males respectively in this reduced population.

In addition, the analyses were rerun using the dose/ response slope (DRS) method of quantifying airway responsiveness [12], lest there had been an important loss of precision because a PD20 of $6,401 \mu \mathrm{g}$ had been assigned to methacholine tests which gave a negative result after the pretreatment phase of the study. The DRS values were skewed, and so their logarithms were used in the further analyses. These produced, reassuringly, identical outcomes to the PD20 analyses. All significant associations between airway responsiveness and fluticasone propionate were confirmed at the same levels of significance, including the treatment/sex interaction, and no new associations were found.

\section{Discussion}

The study confirmed that inhaled fluticasone propionate, at $2,000 \mu \mathrm{g}$ daily, has a beneficial effect on airway responsiveness, but this was no longer evident at the first measurement point 2 weeks after completing the 6-week period of treatment. Consequently no support was found for the notion that intermittent pulsed therapy might be of value as an alternative means of managing mild asthma, or of preventing the emergence of symptoms in subjects with "subclinical" levels of airway responsiveness. This is unfortunate. The recent trend to use inhaled steroids progressively earlier in the management of asthma could have been strengthened if the advantages were achievable with the lesser risks and costs associated with intermittent rather than continuous use.

The geometric mean PD20 increased sequentially and very significantly over 6 weeks as a consequence of fluticasone propionate treatment by 1.9 doublings (3.8-fold) if the sex interaction term is ignored. This takes account of the nonsignificant changes in the placebo group. The use of "fold" increment is not, however, strictly appropriate to PD20 since this is essentially a logarithmic term, but the convention is convenient to clinicians. "Doublings" provides a more acceptable measure of logarithmic change, and is a commonly used alternative term.

The outcome observed may be compared with that of the one other study of similar design, that of OLIVIERI et al. [13]. They observed an equivalent improvement in airway responsiveness of $\sim 2.6$ fold after 6 weeks' fluticasone propionate treatment [13]. Their study focused on the serial examination of constituents of bronchoalveolar lavage fluid in a necessarily smaller number of participants $(n=17)$, and this possibly explains their failure to note an improvement of significant degree earlier in the 6week period. The lesser mean response was probably a consequence of the lesser daily dose of fluticasone propionate $(500 \mu \mathrm{g})$, and this suggests that the beneficial response in airway responsiveness to fluticasone propionate is, not surprisingly, dose-dependent. 
The participants in the present study were selected, perhaps uniquely, to have never previously received corticosteroid treatment nor any other regular treatment for asthma, and so the response observed was not limited in any way by tachyphylaxis. Although the improvement in airway responsiveness at V4 represents the maximum effect of fluticasone propionate treatment during the investigation, there was an important trend of increasing benefit throughout the 6-week treatment period. It is possible, therefore, that further benefit would have occurred had the treatment period been more prolonged.

It is also possible that the censoring effect, by which PD20 were restricted to $6,401 \mu \mathrm{g}$ in five fluticasone propionate treated subjects who gave negative results to the methacholine test at V4, may have led to an underestimation of the true magnitude of the fluticasone propionate effect. However, this underestimation is not likely to have been of major degree since only five of the 23 fluticasone propionate group participants were affected in this way, and by chance they had lower VBPD20 (geometric mean $173 \mu \mathrm{g})$ than the other $18(257 \mu \mathrm{g})$. They consequently contributed more, not less, than average to the overall increase in geometric mean PD20 in the fluticasone propionate group. Furthermore, the DRS analyses of the methacholine tests, which provided a measure of airway responsiveness even when the cumulative decrement in FEV1 failed to reach $20 \%$, gave essentially identical outcomes to those using PD20.

The selection procedure produced a study population with a normal mean baseline level of ventilatory function, and so the failure to observe an increase in FEV1 in the fluticasone propionate group as a consequence of treatment, despite the previous naivety to such treatment, is not unexpected. This usefully confirms that the changes observed in PD20 were fundamentally a consequence of true improvements in airway responsiveness, and not merely a consequence of improvements in ventilatory function.

The sex difference in the response to fluticasone propionate provides an outcome of particular interest. The authors are not aware of any previous reports of sex acting as an effect modifier for corticosteroid therapy in asthma, although preliminary and unpublished results from the European Study of Chronic Obstructive Pulmonary Disease (EUROSCOP) study assessing the effect of inhaled corticosteroids on the progression of chronic obstructive pulmonary disease (COPD) [14] did suggest a sex difference benefiting males. Subsequent analyses have suggested that this was a consequence of confounding, and that an adverse effect of continuing smoking was the true explanation for the apparent sex difference (R. Pauwels, personal communication). A degree of confounding was also feared in the present study (the effect of fluticasone propionate appeared particularly strong in males who were nonsmokers), but, if sex was excluded from the regression analyses, no effect of smoking was detected, nor was there a treatment/smoking interaction. The same outcome was observed from the DRS analyses, and so the results indicate that the effect of fluticasone propionate was modified by sex rather than smoking in the young adult population selected to have PD20 of $<1,600 \mu \mathrm{g}$ (rather than COPD). Thus males appear to be more responsiveness than females, but it may be convenient to consider that a relative failure to respond to inhaled fluticasone propionate is more prevalent in females.
Such effect modification is biologically plausible because a number of recent reports suggest that females affected by asthma (and treated accordingly with inhaled corticosteroids) have more severe disease than males. Not only does a male dominance in asthma prevalence in childhood give way to a mild female dominance in adult life but also adult female asthmatics report greater impairment in quality of life, have a disproportionate number of hospital admissions and accrue greater management costs [15-20]. It has been argued that these differences could be a consequence primarily of subjective differences in perception between the sexes [21, 22], and such differences have been demonstrated by the authors [23], but this does not explain all of the sex effect since there are also reported differences of a more objective nature. For example, Lin and REHMAN [24] noted that of asthmatic subjects with severe exacerbations necessitating intensive care, females required longer periods of intubation than males. Tough et al. [25] noted in males but not females that there was a marked seasonal pattern to asthma mortality, which suggested that summer environmental factors were strong determinants of asthma death in males. During the winter season, when these factors were less influential and male mortality was low, the unchanged (i.e. perennial) level of female mortality was disproportionately high. ROBERTs et al. [26] noted a mortality of 2.25 per 100,000 in 1989 in females compared with 1.38 in males. Mortality data concerning asthma are, however, a further notorious source of inaccuracy [27]. If, nevertheless, there is an important sex anomaly concerning the clinical severity of asthma, the present findings may help to explain it.

It is concluded that, in the absence of any possibility of tachyphylaxis, inhaled fluticasone propionate at $2,000 \mu \mathrm{g}$ daily caused a steadily increasing improvement in airway responsiveness over a 6-week period, which was enhanced by male sex but lost almost immediately on treatment cessation. Short pulses of intermittent prophylactic treatment would not, therefore, be useful as a means of managing mild asthma.

Acknowledgements. The authors are most grateful to M. Abrahamowicz of McGill University, Montreal, for statistical advice and guidance and to G. Devereux who was principally responsible for the initial epidemiological study.

\section{References}

1. Devereux G, Ayatollahi T, Ward R, et al. Airway responsiveness, asthma, and air pollution in two contrasting districts of northern England. Thorax 1996; 51: 169-174.

2. Meibohm B, Mollmann H, Wagner M, Hochhaus G, Mollman A, Derendorf H. The clinical pharmacology of fluticasone propionate. Rev Contemp Pharmacother 1998; 9: 535-549.

3. Weiss ST, Gold DR. Guest editorial: gender differences in asthma. Pediatr Pulmonol 1995; 19: 153-155.

4. Gerritsen J, Koeter G, Postma D, Schouten J, Knol K. Prognosis of asthma from childhood to adulthood. $\mathrm{Am}$ Rev Respir Dis 1989; 140: 1325-1330.

5. Withers NJ, Low L, Holgate ST, Clough JB. The natural history of respiratory symptoms in a cohort of adolescents. Am J Respir Crit Care Med 1998; 158: 352-357.

6. Strachen DP, Cook DG. Health effects of passive smoking. 6. Parental smoking and childhood asthma: 
longitudinal and case control studies. Thorax 1998; 53: 204-212.

7. Beach JR, Young CL, Avery AJ, et al. Measurement of airway responsiveness to methacholine: relative importance of the precision of drug delivery and the method of assessing response. Thorax 1993; 48: 239-243.

8. Jarvis MJ, Tunstall-Pedoe H, Feyerabend C, Vessey C, Saloojee Y. Comparison of tests used to distinguish smokers from nonsmokers. Am J Public Health 1987; 77 : $1435-1438$.

9. Stenton SC, Dennis JH, Walters EH, Hendrick DJ. The asthmagenic properties of a newly developed detergent ingredient - sodium iso-nonanoyl oxybenzene sulphonate. Br J Industr Med 1990; 47: 405-410.

10. Convery RP, Ward RJ, Hendrick DJ. Occupational asthma due to a widely used soft solder flux not containing colophony. Eur Respir J 1997; 10: 238-240.

11. Aiken TC, Ward RJ, Peel ET, Hendrick DJ. Occupational asthma due to porcine pancreatic amylase. Occup Environ Med 1997; 54: 762-764.

12. O'Connor G, Sparrow D, Taylor D, Segal M, Weiss S. Analysis of dose-response curves to methacholine. Am Rev Respir Dis 1987; 136: 1412-1417.

13. Olivieri D, Chetta A, del Donno M, et al. Effect of shortterm treatment with low-dose inhaled fluticasone propionate on airway inflammation and remodelling in mild asthma: a placebo-controlled study. Am J Respir Crit Care Med 1997; 155: 1864-1871.

14. Pauwels RA, Lofdahl CG, Laitinen LA, et al. Treatment with inhaled budesonide in patients with mild chronic obstructive pulmonary disease who continue smoking. European Respiratory Society Study on Chronic Obstructive Pulmonary Disease. N Engl J Med 1999; 340: 1948-1953.

15. Togias A, Horowitz E, Joyner D, Guydon L, Malveaux F. Evaluating the factors that relate to asthma severity in adolescents. Int Arch Allergy Immunol 1997; 113: 87-95.

16. Sunyer J, Anó JM, Kogevinas M, et al. Risk factors for asthma in young adults. Eur Respir $J$ 1997; 10: 2490 2494.
17. Bousquet J, Knani J, Dhivert $\mathrm{H}$, et al. Quality of life in asthma. 1. Internal consistency and validity of the SF-36 questionnaire. Am J Respir Crit Care Med 1994; 149: 371-375.

18. Skobeloff EM, Spivey WH, St Clair SS, Schoffstall JM. The influence of age and sex on asthma admissions. JAMA 1992; 268: 3437-3440.

19. Otopade CO, Alikakos Z, Abubaker J, Rubinstein IR. Characteristics of predominantly nonwhite patients with frequent hospitalizations for acute asthma in Chicago. $J$ Asthma 1997; 34: 243-248.

20. Stempl DA, Hedblom C, Durcanin-Robbins JF, Sturm LL. Use of a pharmacy and medical claims database to document cost centers for 1993 annual asthma expenditure. Arch Fam Med 1996; 36: 36-40.

21. Prescott E, Lange P, Vestbo J. Effect of gender on hospital admissions for asthma and prevalence of self-reported asthma: a prospective study based on a sample of the general population. Thorax 1997; 52: 287-289.

22. Osborne ML, Vollmer WM, Linton KLP, Buist AS. Characteristics of patients with asthma within a large HMO. Am J Respir Crit Care Med 1998; 157: 123-128.

23. Devereux G, Hendrick DJ, Stenton SC. Perception of respiratory symptoms after methacholine-induced bronchoconstriction in a general population. Eur Respir J 1998; 12: 1089-1093.

24. Lin RY, Rehman A. Clinical characteristics of adult asthmatics requiring intubation. $J$ Med 1995; 26: 261-277.

25. Tough SC, Green FH, Paul JE, Wigle DT, Butt JC. Sudden death from asthma in 108 children and young adults. J Asthma 1996; 33: 179-188.

26. Roberts C, Mayer JD, Henderson WR. Asthma deaths in Washington State, 1980-1989 geographic and demographic distributions. Ann Allergy Asthma Immunol 1996; 76: $20-26$

27. Reid DWEC, Hendrick VJ, Aiken TC, Berrill WT, Stenton SC, Hendrick DJ. Age-dependent inaccuracy of asthma death certification in Northern England, 19911992. Eur Respir J 1998; 12: 1079-1083. 